\title{
Deep Colorization for Surveillance Images
}

\author{
Chen $\mathrm{Yao}^{1,2}$ and Yan Xia ${ }^{1}$ \\ ${ }^{1}$ The Third Research Institute of Ministry of Public Security, Shanghai, China \\ ${ }^{2}$ Shanghai Key Laboratory of Digital Media Processing and Transmission
}

\begin{abstract}
In video surveillance application, grayscale image often influences the image processing results. In order to solve the colorization problem for surveillance images, this paper propose a fully end-to-end approach to obtain a reasonable colorization results. A CNN learning structure and gradient prior are be used for chromatic space inferring. Finally, our experimental results show our advantage.
\end{abstract}

\section{Introduction}

Image colorization is the process of adding colour to grayscale image. Because of lacking of prior colour information, image colorization is an ill-posed problem which is intractable to solve. And, colour propagation across different image semantic region is often produced during image colorization. How to obtain a reasonable colour for a grayscale image is a challenging problem. For the image surveillance application, a lot of network cameras produce many grayscale images, which greatly affect image application such as tracking, recognition etc. Traditional colorization approaches include scribblebased and non-scribble based way. In scribble-based methods, many strategies are proposed such as optimization-based, geodesic distance based, texture similarity based and texture classification colorization etc. [1-10] Non-scribble colorization often utilizes colours of reference image and transfers the target colour to input image. For this way, colour mapping functions between reference image and target image is computed. Moreover, supervised learning scheme is also exploited for predict low-level features. In this paper, our algorithm is implemented on Lab colour space. For a given illumination, we try to infer the $\mathrm{a}$ and $\mathrm{b}$ colour channels of input images based on a convolutional neural networks. [11-13]

In the rest part of this paper, $\mathrm{CNN}$-based colorization scheme will be introduced in section 2. Experimental results are provided in section 3 . And finally section 4 concludes the paper.

\section{Proposed algorithm}

We use a VGG network with repeated conv, ReLU and BatchNorm layers. In our algorithm, pooling is not adopted in the network. Image resolution transformation is obtained by downsampling and upsampling. Inspired by Zhang's method [9], we build a more reasonable object function to getting plausible colours. For a given illumination L, we try to learn a mapping $\hat{I}=\mathrm{F}(L), \hat{I}$ represents the colour probability distribution in ab colour space. Here, a cross entropy loss function is provided as following,

$$
\operatorname{Loss}(\hat{I}, I)=-\sum W \sum I \cdot \log (I)
$$

Where, $\mathrm{W}$ is a weighting coefficients of gradient probability distribution. A Gaussian kernel is used for computing final weighting coefficients. After we get the colour probability distribution by an end-to-end training, we use a point estimation to obtain final colour value $\mathrm{C}$ as following,

$$
C_{\mathrm{a}, \mathrm{b}}=\frac{\exp (\log (I) / V)}{\sum \exp (\log (I) / V)}
$$

Where, $\mathrm{V}$ is regularization controlling parameter.

\section{Experiments}

In this section, different colorization results are demonstrated. Our training database is close to $1 \mathrm{M}$ surveillance images.

Figure 1 shows a colorization example using our algorithm. Figure1(a) and Figure 1(b) are original grayscale images. And, Figure 1(c) and Figure 1(d) are the colorization results. It can be seen that our method performs a good colorization, which closes to real surveillance scene. Figure 2 is another example, which also illustrate our advantage. In our last example, school bus and kindergarten are shown as following. From all of the experimental results, the colour of results can not show variability comparing with real application. This will be solved in our future work. 


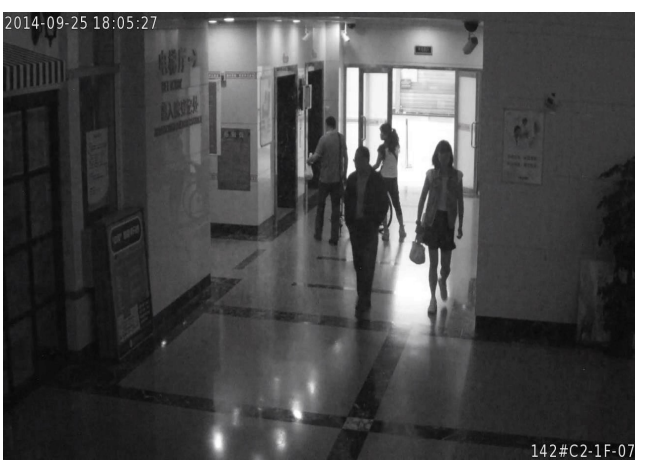

(a)

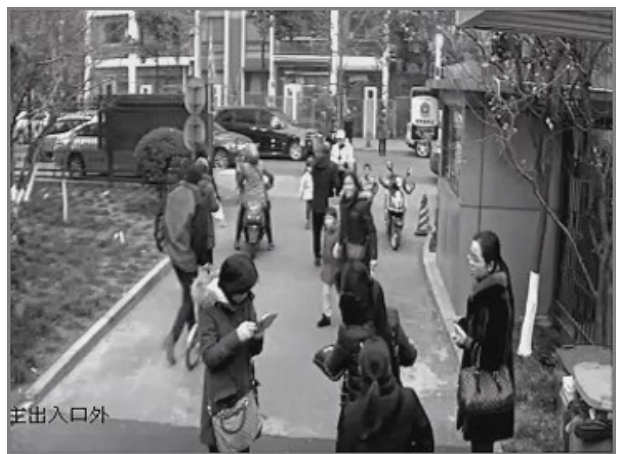

(b)

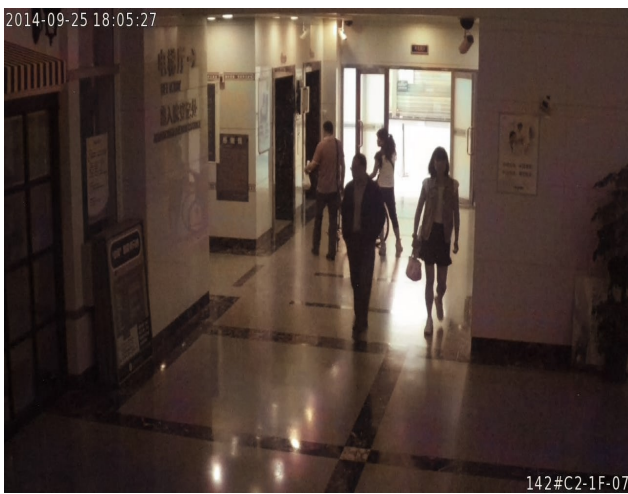

(c)

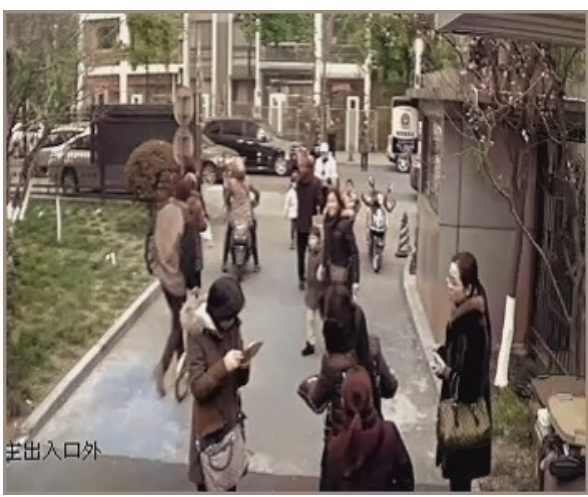

(d)

Figure 1. The effect of colorization for surveillance images. a and $\mathrm{b}$ are grayscale images, $\mathrm{c}$ and $\mathrm{d}$ are colorization results.

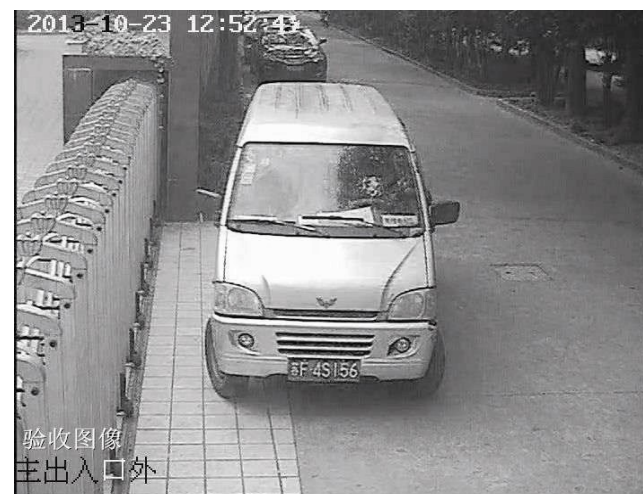

(a)

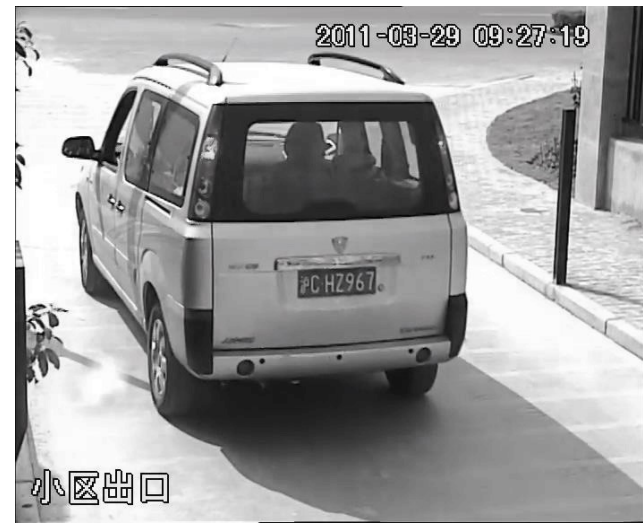

(b)

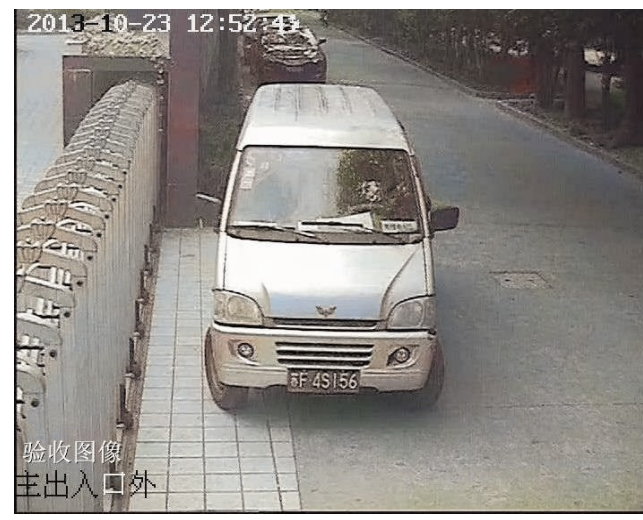

(c)

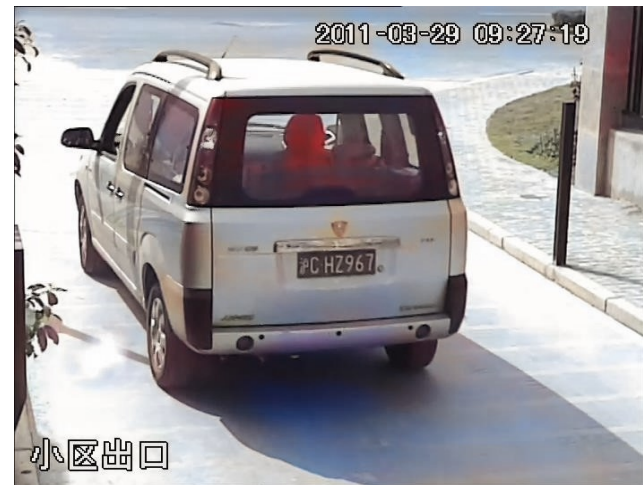

(d)

Figure 2. The effect of colorization for surveillance images. a and $\mathrm{b}$ are grayscale images, $\mathrm{c}$ and $\mathrm{d}$ are colorization results. 


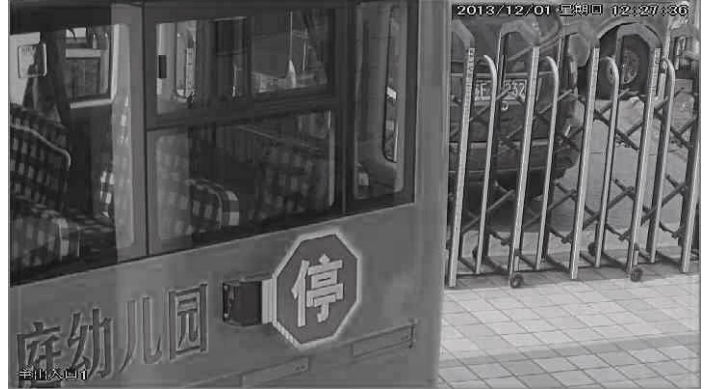

(a)

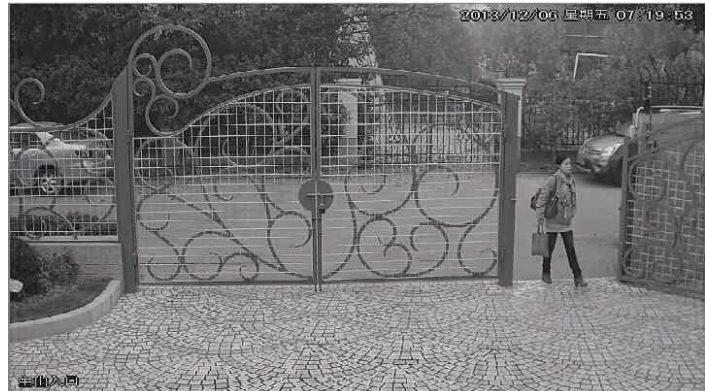

(b)

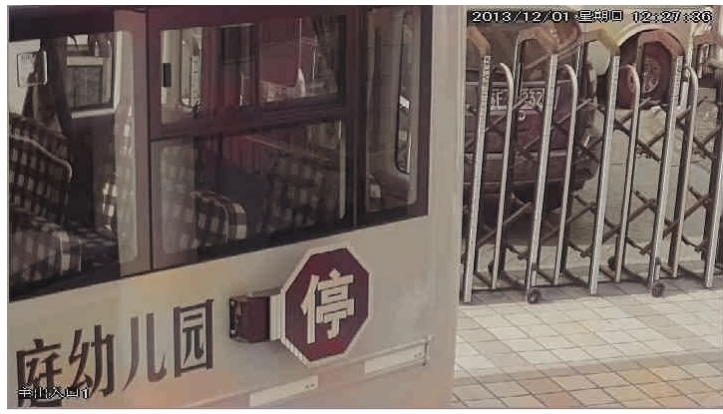

(c)

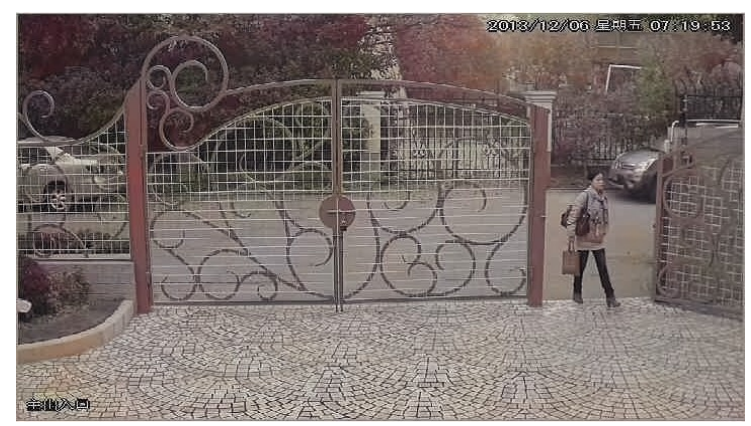

(d)

Figure 3. The effect of colorization for surveillance images. a and $\mathrm{b}$ are grayscale images, $\mathrm{c}$ and $\mathrm{d}$ are colorization results.

\section{Conclusions}

In this paper, we presented a CNN-based self-supervised colorization method inspired by gradient prior fused endto-end training. Experiment results demonstrate the benefits of colour probability weighting colorization.

\section{Acknowledgement}

This work was supported by the Opening Project of Shanghai Key Laboratory of Digital Media Processing and Transmission.

\section{References}

1. LEVIN, A., LISCHINSKI, D., AND WEISS, Y. 2004. Colorization using optimization. ACM Transactions on Graphics 23, 689-694.

2. HUANG, Y.-C., TUNG, Y.-S., CHEN, J.-C., WANG, S.-W., AND WU, J.-L. 2005. An adaptive edge detection based colorization algorithm and its applications. In ACM International Conference on Multimedia, 351-354.

3. Cheng, Z., Yang, Q., Sheng, B.: Deep colorization. In: Proceedings of the IEEE International Conference on Computer Vision. (2015) 415-423.

4. Dahl, R.: Automatic colorization. In: http://tinyclouds.org/colorize/. (2016)

5. $\mathrm{G}$ Charpiat, $\mathrm{M}$ Hofmann, $\mathrm{B}$ Schölkopf, B.: Automatic image colorization via multimodal predictions. In: Computer Vision \{ECCV 2008. Springer (2008) 126-139.

6. Ramanarayanan, G., Ferwerda, J., Walter, B., Bala, K.: Visual equivalence: towards a new standard for image delity. ACM Transactions on Graphics (TOG) 26 (3) (2007).

7. Simonyan, K., Zisserman, A.: Very deep convolutional networks for large-scale image recognition. arXiv preprint arXiv:1409.1556 (2014)

8. TAI, Y., JIA, J., AND TANG, C. 2005. Local color transfer via probabilistic segmentation by expectation-maximization. In CVPR, 747-754.

9. Zhang $\mathrm{R}$, Isola $\mathrm{P}$, Efros $\mathrm{A} \mathrm{A}$. Colorful image colorization [C]. European Conference on Computer Vision. Springer, Cham, 2016: 649-666.

10. WANG, X., FOUHEY, D. F., AND GUPTA, A. 2015. Designing deep networks for surface normal estimation. In CVPR.

11. WELSH, T., ASHIKHMIN, M., AND MUELLER, K. 2002. Transferring color to greyscale images. ACM Trans. Graph. 21, 3(July), 277-280.

12. WU, F., DONG, W., KONG, Y., MEI, X., PAUL, J.C., AND ZHANG, X. 2013. Content-based colour transfer. Computer Graphics Forum 32, 1, 190-203.

13. XU, K., LI, Y., JU, T., HU, S.-M., AND LIU, T.-Q. 2009. Efficient affinity-based edit propagation using k-d tree. ACM Trans. Graph. 28, 5 (Dec.), 118: 1118: 6 . 